\title{
HILBERT TRANSFORM ASSOCIATED WITH FINITE MAXIMAL SUBDIAGONAL ALGEBRAS
}

\author{
NARCISSE RANDRIANANTOANINA
}

(Received 30 January 1998; revised 17 August 1998)

\author{
Communicated by P. G. Dodds
}

\begin{abstract}
Let $\mathscr{M}$ be a von Neumann algebra with a faithful normal trace $\tau$, and let $H^{x}$ be a finite, maximal. subdiagonal algebra of $\mathscr{M}$. We prove that the Hilbert transform associated with $H^{x}$ is a linear continuous map from $L^{1}(\mathscr{M}, \tau)$ into $L^{1, x}(\mathscr{M}, \tau)$. This provides a non-commutative version of a classical theorem of Kolmogorov on weak type boundedness of the Hilbert transform. We also show that if a positive measurable operator $b$ is such that $b \log ^{+} b \in L^{\prime}(\mathscr{M}, \tau)$ then its conjugate $\tilde{b}$, relative to $H^{x}$ belongs to $L^{1}(\mathscr{M}, \tau)$. These results generalize classical facts from function algebra theory to a non-commutative setting.
\end{abstract}

1991 Mathematics subject classification (Amer. Math. Soc.): 46L50, 46E15; Secondary: 43A15, 47D15. Keywords and phrases: von-Neumann algebras, conjugate functions, Hardy spaces.

\section{Introduction}

The theory of conjugate functions has been a strong motivating force behind various aspects of harmonic analysis and abstract analytic function spaces. This theory which was originally developed for functions in the circle group $\mathbb{T}$ has found many generalizations to more abstract settings of function algebras such as Dirichlet algebras in [6] and weak*-Dirichlet algebras in [15]. Results from this theory have been proven to be very fruitful for studying Banach space properties of the Hardy spaces (and their relatives) associated with the algebra involved (see for instance [3] and [17]).

The notion of conjugate operators, Hilbert transforms along with Riesz projections for non-commutative settings have been considered by several authors. For instance, one can find implicitly in a paper of Zsido [30] that for $1<p<\infty$, the Hilbert transform is a bounded linear map on non-commutative $L^{p}$ associated with a general

(C) 1998 Australian Mathematical Society 0263-6115/98 \$A2.00+0.00 
semifinite von Neumann algebra. Zsido's approach was to study representations of locally compact groups of operators. The same line of ideas were used by Berkson $e t$ al [13] and Asmar et al [2] to obtain some versions of Riesz projection boundedness for the case $1<p<\infty$. Very recently Dodds et al [11] also considered Riesz projection for the case of symmetric spaces of measurable operators affiliated with semifinite von Neumann algebras.

The main purpose of the present paper is to investigate the Hilbert transform for the case $p=1$. It is well known that the classical Hilbert transform is of weak type 1-1 that is a continuous linear map from $L^{1}(\mathbb{T})$ into the space $L^{1 . \infty}(\mathbb{T})$. This can be obtained from the so called Kolmogorov inequality. This result was proved to be valid for the case of weak*-Dirichlet algebra by Hirschman and Rochberg [15]. A question that arises naturally from these facts is whether or not a non-commutative analogue of Kolmogorov's theorem is valid. Let $\mathscr{M}$ be a von Neumann algebra with a faithful, normal finite trace $\tau$. Arveson [1] introduced, as non-commutative analogues of weak*-Dirichlet algebras, the notion of finite, maximal subdiagonal algebras of $\mathscr{M}$ (see definition below). Subsequently several authors studied the (non-commutative) $H^{p}$-spaces associated with such algebras (see for instance, $[16,20,22,24,25]$ ).

We prove that most fundamental theorems on conjugate operation on Hardy spaces associated with weak* ${ }^{*}$ Dirichlet (see [6] and [15]) remain valid for Hardy spaces associated with finite subdiagonal algebras. In particular, we show that the conjugation operator is a continuous linear map from $L^{1}(\mathscr{M}, \tau)$ into $L^{1 . \infty}(\mathscr{M}, \tau)$. This allows us to conclude that, as in the commutative case, the Hilbert transform is bounded from $L^{\prime}(\mathscr{M}, \tau)$ into $L^{p}(\mathscr{M}, \tau)$ for $0<p<1$.

We refer to $[23,26]$ and [27] for general information concerning von Neumann algebras as well as basic notions of non-commutative integration, to [7] and [19] for Banach space theory and to [14] and [31] for basic definitions from harmonic analysis.

\section{Definitions and some preliminary results}

Throughout, $H$ will denote a Hilbert space and $\mathscr{M} \subseteq \mathscr{L}(H)$ a von Neumann algebra with a normal, faithful finite trace $\tau$. A closed densely defined operator $a$ in $H$ is said to be affiliated with $\mathscr{M}$ if $u^{*} a u=a$ for all unitary operators $u$ in the commutant $\mathscr{M}^{\prime}$ of $\mathscr{M}$. If $a$ is a densely defined self-adjoint operator on $H$, and if $a=\int_{-\infty}^{\infty} s d e_{s}^{a}$ is its spectral decomposition, then for any Borel subset $B \subseteq \mathbb{R}$, we denote by $\chi_{B}(a)$ the corresponding spectral projection $\int_{-\infty}^{\infty} \chi_{B}(s) d e_{s}^{a}$. A closed densely defined operator $a$ on $H$ affiliated with $\mathscr{M}$ is said to be $\tau$-measurable if there exists a number $s \geq 0$ such that $\tau\left(\chi_{(s, \infty)}(|a|)\right)<\infty$.

The set of all $\tau$-measurable operators will be denoted by $\overline{\mathscr{M}}$. The set $\overline{\mathscr{M}}$ is a *-algebra with respect to the strong sum, the strong product, and the adjoint operation 
[23]. For $x \in \overline{\mathscr{M}}$, the generalized singular value function $\mu(x)$ of $x$ is defined by

$$
\mu_{t}(x)=\inf \left\{s \geq 0: \tau\left(\chi_{(s . \infty)}(|x|)\right) \leq t\right\}, \quad \text { for } t \geq 0 .
$$

The function $t \rightarrow \mu_{t}(x)$ from $(0, \tau(I))$ to $[0, \infty)$ is right continuous, non-increasing and is the inverse of the distribution function $\lambda(x)$, where $\lambda_{s}(x)=\tau\left(\chi_{\mid s, x)}(|x|)\right)$, for $s \geq 0$. For some basic properties of $\mu($.$) and \lambda($.$) , we refer to [12]; some additional$ properties can be found in [8] and [10]. Using the generalized singular value function, one can construct a non-commutative version of a given function space as follows.

DEFINITION 1. Let $E$ be an order continuous rearrangement invariant (quasi-) Banach function space on $(0, \tau(I))$. We define the symmetric space $E(\mathscr{M}, \tau)$ of measurable operators by setting:

$$
\begin{aligned}
& E(\mathscr{M}, \tau)=\{x \in \overline{\mathscr{M}}: \mu(x) \in E\} \quad \text { and } \\
& \|x\|_{E(\mathscr{M}, \tau)}=\|\mu(x)\|_{E}, \text { for } x \in E(\mathscr{M}, \tau) .
\end{aligned}
$$

It is well known that $E(\mathscr{M}, \tau)$ is a Banach space (respectively, quasi-Banach space) if $E$ is a Banach space (respectively, quasi-Banach space), and that if $E=L^{p}(0, \tau(I))$, for $0<p<\infty$, then $E(\mathscr{M}, \tau)$ coincides with the usual non-commutative $L^{p}$-space associated with $(\mathscr{M}, \tau)$. We refer to $[4,8]$ and $[28]$ for more detailed discussions about these spaces. For simplicity we will always assume that the trace $\tau$ is normalized.

The next definition isolates the main topic of this paper.

DEFINITION 2. Let $H^{\infty}$ be a weak-closed unital subalgebra of $\mathscr{M}$ and let $\Phi$ be a faithful, normal expectation from $\mathscr{M}$ onto the diagonal $D=H^{\infty} \cap\left(H^{\infty}\right)^{*}$, where $\left(H^{\infty}\right)^{*}=\left\{x^{*}, x \in H^{\infty}\right\}$. Then $H^{\infty}$ is called a finite, maximal, subdiagonal algebra in $\mathscr{M}$ with respect to $\Phi$ and $\tau$ if:

(1) $H^{\infty}+\left(H^{\infty}\right)^{*}$ is weak ${ }^{*}$-dense in $\mathscr{M}$;

(2) $\Phi(a b)=\Phi(a) \Phi(b)$ for all $a, b \in H^{\infty}$;

(3) $H^{\infty}$ is maximal among those subalgebras satisfying (1) and (2);

(4) $\tau \circ \Phi=\tau$.

For $0<p<\infty$, the closure of $H^{\infty}$ in $L^{p}(\mathscr{M}, \tau)$ is denoted by $H^{p}(\mathscr{M}, \tau)$ (or simply $H^{p}$ ) and is called the Hardy space associated with the subdiagonal algebra $H^{\infty}$. Similarly, the closure of $H_{0}^{\infty}=\left\{x \in H^{\infty} ; \Phi(x)=0\right\}$ is denoted by $H_{0}^{p}$. These non-commutative Hardy spaces were first considered by Arverson [1] and have been studied by several authors.

Note that the conditional expectation $\Phi$ extends to $L^{2}(\mathscr{M}, \tau)$ and this extension is an orthogonal projection from $L^{2}(\mathscr{M}, \tau)$ onto $[D]_{2}$, the closure of $D$ in $L^{2}(\mathscr{M}, \tau)$. Similarly, since $\|\Phi(x)\|_{1} \leq\|x\|_{1}$ for every $x \in \mathscr{M}$, the operator $\Phi$ extends uniquely to a projection of norm one from $L^{1}(\mathscr{M}, \tau)$ onto $[D]_{1}$, the closure of $D$ in $L^{1}(\mathscr{M}, \tau)$. 
Let $\mathscr{A}=H^{x}+\left(H^{x}\right)^{*}$. Since $\mathscr{A}$ is weak ${ }^{*}$-dense in $\mathscr{M}$, it is norm dense in $L^{p}(\mathscr{M}, \tau)$, where $1 \leq p<\infty$.

Note that $H^{\infty}$ and $\left(H_{0}^{\times}\right)^{*}$ are orthogonal in $L^{2}(\mathscr{M}, \tau)$. This fact implies that $L^{2}(\mathscr{M}, \tau)=H^{2} \oplus\left(H_{0}^{2}\right)^{*}$, and hence that $L^{2}(\mathscr{M}, \tau)=H_{0}^{2} \oplus\left(H_{0}^{2}\right)^{*} \oplus[D]_{2}$.

Let $a \in \mathscr{A}$. Then $a$ can be written as $a_{1}+a_{2}^{*}+d$ where $a_{1}$ and $a_{2}$ belong to $H_{0}^{x}$ and $d \in D$. In fact, $a=b_{1}+b_{2}^{*}$ with $b_{1}, b_{2} \in H^{\infty}$ and set $d=\Phi\left(b_{1}\right)+\Phi\left(b_{2}^{*}\right) \in D$ and $a_{i}=b_{i}-\Phi\left(b_{i}\right)$, for $i=1,2$.

Since $H_{0}{ }^{\infty}$ and $\left(H_{0}^{\infty}\right)^{*}$ are orthogonal subsets of $L^{2}(\mathscr{M}, \tau)$, this decomposition is unique.

DEFINITION 3. For $u=u_{1}+u_{2}^{*}+d$ in $\mathscr{A}$, we define the conjugate of $u$ by setting $\tilde{u}=i u_{2}^{*}-i u_{1}$.

It is clear that for every $u \in \mathscr{A}$, the operator $\tilde{u} \in \mathscr{M}$ and $u+i \tilde{u}=2 u_{1}+d \in H^{\infty}$. The Hilbert transform (conjugation operator) $\mathscr{H}$ can now be defined as a map on $\mathscr{A}$ as follows: $\mathscr{H}: \mathscr{A} \rightarrow \mathscr{M}(u \rightarrow \mathscr{H}(u)=\tilde{u})$. For more general setting of conjugate operators and Hilbert transform, we refer to [30]. It should be noted that if $\mathscr{M}$ is commutative, then the above definition coincides with the definition of conjugate functions for weak*-Dirichlet algebras studied in [15].

REMARKS 1. (i) If $u=u^{*}$, then the uniqueness of the decomposition implies that $u_{1}=u_{2}$ and $d=d^{*}$. Therefore if $u=u^{*}$ then $\tilde{u}=\tilde{u}^{*}$.

(ii) For $u=u_{1}+u_{2}^{*}+d \in \mathscr{A}$ and $\tilde{u}=i\left(u_{2}^{*}-u_{1}\right)$, the above observation implies that $u_{2}^{*} \perp u_{1}$ in $L^{2}(\mathscr{M}, \tau)$, so

$$
\|\tilde{u}\|_{2}^{2}=\left\|u_{2}^{*}-u_{1}\right\|_{2}^{2}=\left\|u_{2}^{*}\right\|_{2}^{2}+\left\|u_{1}\right\|_{2}^{2} .
$$

and since $L^{2}(\mathscr{M}, \tau)=H_{0}^{2} \oplus\left(H_{0}^{2}\right)^{*} \oplus[D]_{2}$ we get,

$$
\|u\|_{2}^{2}=\left\|u_{1}\right\|_{2}^{2}+\left\|u_{2}^{*}\right\|_{2}^{2}+\|d\|_{2}^{2},
$$

which implies that $\|\tilde{u}\|_{2} \leq\|u\|_{2}$

As a consequence of (ii). it is clear that $\mathscr{H}$ can be extended as a bounded map of norm one on $L^{2}(\mathscr{M}, \tau)$. In fact the following more general result can be deduced from [30]:

THEOREM 1. For each $1<p<\infty$, there is a unique continuous linear extension of $\mathscr{H}$ (which is also denoted by $\mathscr{H}$ ) from $L^{p}(\mathscr{M}, \tau)$ into $L^{p}(\mathscr{M}, \tau)$ with the property that $f+i \tilde{f} \in H^{n}$ for all $f \in L^{\prime \prime}(\mathscr{M}, \tau)$. Moreover there is a constant $C_{p}=8 \max (p, q)$ such that

$$
\|\tilde{f}\|_{p} \leq C_{p}\|f\|_{p} \quad \text { for all } f \in L^{p}(\mathscr{M}, \tau) \text { and } 1 / p+1 / q=1 .
$$


We present a short discussion on how the constant $C_{p}$ is obtained. For $k \in \mathbb{N}$ and $p=2 k$, the above theorem can be easily obtained from [30, Lemma 4.1] replacing $L^{2 k}(\rho)^{U^{2}}(E /(-E))$ by $H^{2 k}$; all the assumptions on [30| would be satisfied by our setting.

Let $f=f^{*} \in \mathscr{A}$; from Remarks $1, f=u+u^{*}+d=(u+d / 2)+(u+d / 2)^{*}$ for some $u \in H_{0}^{\infty}$ and $d \in D$ so from [30, Lemma 4.1], we get

$$
\|\tilde{f}\|_{2 k}=\left\|u-u^{*}\right\|_{2 k} \leq 4 k\|f\|_{2 k} .
$$

So for $f \in \mathscr{A}$ (not necessarily self-adjoint),

$$
\|\tilde{f}\|_{2 k} \leq 8 k\|f\|_{2 k} .
$$

Since $\mathscr{A}$ is dense in $L^{2 k}(\mathscr{H}, \tau)$, the inequality above shows that $\mathscr{H}$ can be extended as a bounded linear operator from $L^{2 k}(\mathscr{M}, \tau)$ into $L^{2 k}(\mathscr{M} . \tau)$ with $\|\mathscr{H}\| \leq 8 k$; so the theorem is proved for $p$ even.

For the general case, let $2 \leq p<\infty$. Choose an integer $k$ such that $2 k \leq p \leq$ $2 k+2$. By [9, Theorem 2.3], $L^{p}(\mathscr{M}, \tau)$ can be realized as a complex interpolation of the pair of Banach spaces $\left(L^{2 k}(\mathscr{M}, \tau), L^{2 k+2}(\mathscr{M}, \tau)\right)$, and we conclude that $\mathscr{H}$ is also bounded from $L^{p}(\mathscr{M}, \tau)$ into $L^{p}(\mathscr{M}, \tau)$ with $\|\mathscr{H}\| \leq(8 k)^{H}(8 k+8)^{1-H}$ for some $0<\theta<1$. Therefore $\|\mathscr{H}\| \leq(8 k+8) \leq 8 p$. So the theorem is verified for $2 \leq p$.

For $1<p<2$, from the above case, $\mathscr{H}$ is bounded from $L^{q}(\mathscr{M}, \tau)$ into $L^{q}(\mathscr{M}, \tau)$, where $1 / p+1 / q=1$, and as in the commutative case. $(\mathscr{H})^{*}=-\mathscr{H}$ and hence $\|\mathscr{H}\| \leq 8 q$. In fact, let $u$ and $v$ be self-adjoint elements of $\mathscr{A}$; we have

$$
\Phi((u+i \tilde{u})(v+i \tilde{v}))=\Phi(u+i \tilde{u}) \Phi(v+i \tilde{v})=\Phi(u) \Phi(v) .
$$

which implies that

$$
\Phi(u v+i u \tilde{v}+i \tilde{u} v-\tilde{u} \tilde{v})=\Phi(u v-\tilde{u} \tilde{v})+i \Phi(u \tilde{v}+\tilde{u} v)=\Phi(u) \Phi(v) .
$$

so

$$
\tau(u v-\tilde{u} \tilde{v})+i \tau(u \tilde{v}+\tilde{u} v)=\tau(\Phi(u) \Phi(v)) .
$$

Since $\Phi(u)$ and $\Phi(v)$ are self-adjoint, $\tau(\Phi(u) \Phi(v)) \in \mathbb{R}$, and also $\tau(u v-\tilde{u} \tilde{v})$ and $\tau(u \tilde{v}+\tilde{u} v) \in \mathbb{R}$. This implies $\tau(u \tilde{v}+\tilde{u} v)=0$ and $\tau(u \tilde{v})=-\tau(\tilde{u} v)$.

REMARKS 2. (i) The above theorem yields that, as in [15, Corollary $2 \mathrm{~h}]$, there exists a constant $M$ such that if $1<p<\infty$ and $1 / p+1 / q=1$, then $\|\tilde{u}\|_{p} \leq$ $M p q\|u\|_{p}$, for all $u \in L^{p}(\mathscr{M}, \tau)$.

(ii) After this paper was written, we also learned that Marsalli and West [21. Theorem 5.5] also considered the Riesz projection for the setting of this paper and provided a direct proof for the above theorem. 
We finish this section by collecting a few lemmas that are necessary for the proof of our main result. The first lemma is just a notational adjustment of [15, Theorem 3a] so we will not present its proof.

Lemma 1. For $u \in \mathscr{M}$. let $f=u+i \tilde{u}$ and $0<\varepsilon<1$. The formal series $\varphi(t)=\sum_{k=1}^{x} t^{k} \varepsilon^{k} f^{k} / k$ ! is absolutely convergent in $H^{1}$ for every $t<1 / e M_{1}\|u\|$ where $M_{1}=2 M+2$ and $M$ is the constant from Remarks 1 (i) above.

Lemma 2. For $u \in \mathscr{M}, u \geq 0$, let $f=u+i \tilde{u}$ and $0<\varepsilon<1$. Then $I+\varepsilon f$ has bounded inverse with $\left\|(I+\varepsilon f)^{-1}\right\| \leq 1$ and $f_{\varepsilon}=(\varepsilon I+f)(I+\varepsilon f)^{-1} \in H^{\infty}$.

PrOOF. Note that $f$ is densely defined and that, for every $x \in D(f)$,

$$
\langle(I+\varepsilon f) x, x\rangle=\langle(I+\varepsilon u) x, x\rangle+i\langle\tilde{u} x, x\rangle .
$$

Thus $|\langle(I+\varepsilon f) x, x\rangle| \geq\|x\|^{2}$, which implies $\|(I+\varepsilon f) x\| \geq\|x\|$ for all $x \in D(f)$. So $I+\varepsilon f$ has bounded inverse with $\left\|(I+\varepsilon f)^{-1}\right\| \leq 1$.

For the second part, note first that $f_{\varepsilon}$ is bounded. In fact, $f_{\varepsilon}=\varepsilon(I+\varepsilon f)^{-1}+f(I+$ $\varepsilon f)^{-1}$; but $I=(I+\varepsilon f)(I+\varepsilon f)^{-1}=(I+\varepsilon f)^{-1}+\varepsilon f(I+\varepsilon f)^{-1}$ and $(I+\varepsilon f)^{-1} \in \mathscr{M}$, so the operator $\varepsilon f(I+\varepsilon f)^{-1}$ is bounded, implying $f(I+\varepsilon f)^{-1}$ is bounded. To prove that $f_{\varepsilon} \in H^{x}$, it suffices to show that $(I+\varepsilon f)^{-1} \in H^{x}$.

Set $A=-\varepsilon f$. There exists a (unique) semi-group of contractions $\left(T_{t}\right)_{t>0}$ such that $A$ is the infinitesimal generator of $\left(T_{t}\right)_{t>0}$ (see for instance [29, p. 246-249]). It is well known that

$$
\begin{aligned}
& (I-A)^{-1} x=\int_{0}^{\infty} e^{-t} T_{t} x d t \quad \text { for all } x \in H \quad \text { and } \\
& T_{t} x=\lim _{n \rightarrow \imath} \exp \left(t A\left(I-n^{-1} A\right)^{-1}\right) x \quad \text { for all } x \in H .
\end{aligned}
$$

It is claimed that $T_{t} \in H^{x}$ for every $t>0$.

Since $\left(T_{t}\right)_{t>0}$ is a semi-group and $H^{x}$ is a subalgebra, it is enough to verify this claim for small values of $t$. Assume that $2 t \leq 1 / e M_{1}\|u\|$. Let $\varphi(t)$ be the operator in $H^{1}$ defined in Lemma 1 . We will show that $T_{t}=\varphi(t)$. Using the series expansion of the exponential and Lemma 1 , we get

$$
\begin{aligned}
\left\|\exp \left(t A\left(I-n^{-1} A\right)^{-1}\right)-\varphi(t)\right\|_{1} & \leq \sum_{k \geq 0} \frac{t^{k}}{k !}\left\|\left(A\left(I-n^{-1} A\right)^{-1}\right)^{k}-A^{k}\right\|_{1} \\
& \leq \sum_{k \geq 0}\left\|\frac{(2 t)^{k}}{k !} \varepsilon^{k} f^{k}\right\|_{1}<\infty .
\end{aligned}
$$

Fix $k \geq 0$ and set for every $n \geq 1, J_{n}=\left(I-n^{-1} A\right)^{-1}$.

$$
\left\|\left(A J_{n}\right)^{k}-A^{k}\right\|_{1}=\left\|A^{k}\left(J_{n}\right)^{k}-A^{k}\right\|_{1} \leq\left\|A^{k}\right\|_{2}\left\|\left(J_{n}\right)^{k}-I\right\|_{2} .
$$


Since $J_{n} x-x=n^{-1} J_{n} A x$ for every $x \in D(A)$ (see for instance [29, p. 246]), one can conclude that $\left\|J_{n}-I\right\|_{1}$ converges to zero. Similarily, $\left\|\left(J_{n}\right)^{k}-I\right\|_{2}$ converges to zero which implies that $\lim _{n \rightarrow \infty}\left(t^{k} / k !\right)\left\|\left(A\left(I-n^{-1} A\right)^{-1}\right)^{k}-A^{k}\right\|_{1}=0$ so by the estimate on the series above,

$$
\lim _{n \rightarrow \infty}\left\|\exp \left(t A\left(I-n^{-1} A\right)^{-1}\right)-\varphi(t)\right\|_{1}=0
$$

which shows that $T_{t}=\varphi(t) \in H^{1}$ and since $T_{t}$ is bounded, the claim follows.

We conclude the proof by noticing that $t \rightarrow T_{t}$ is a continuous function in $H^{1}$ which shows that $(I-A)^{-1} \in H^{1}$.

LEMMA 3. (1) $\Phi\left(f_{\varepsilon}\right)=\Phi(u)_{\varepsilon}$.

(2) $\operatorname{Re}\left(f_{\varepsilon}\right) \geq \varepsilon I$.

(3) $\operatorname{Re}\left(I+\left(f_{\varepsilon}-s I\right)\left(f_{\varepsilon}+s I\right)^{-1}\right) \geq 0$ for every $s>0$.

(4) $\lim _{\varepsilon \rightarrow 0}\left\|f_{\varepsilon}-f\right\|_{p}=0 \quad 1 \leq p<\infty$.

PROOF. (1) $f_{\varepsilon} \in H^{\infty}$ and $f_{\varepsilon}(I+\varepsilon f)=\varepsilon I+f \in H^{2}$, so $\Phi\left(f_{\varepsilon}\right) \Phi(I+\varepsilon f)=$ $\Phi(\varepsilon I+f)$. But $\Phi(f)=\Phi(u)$, so we get $\Phi\left(f_{\varepsilon}\right)(I+\varepsilon \Phi(u))=\varepsilon I+\Phi(u)$.

(2) From the definition of $f_{\varepsilon}$,

$$
\begin{aligned}
\operatorname{Re}\left(f_{\varepsilon}\right) & =\operatorname{Re}\left((\varepsilon I+f)(I+\varepsilon f)^{-1}\right) \\
& \left.=\operatorname{Re}\left(\left[\left(\varepsilon I+\varepsilon^{2} f\right)+\left(1-\varepsilon^{2}\right) f\right)\right](I+\varepsilon f)^{-1}\right) \\
& =\varepsilon I+\left(1-\varepsilon^{2}\right) \operatorname{Re}\left(f(I+\varepsilon f)^{-!}\right) .
\end{aligned}
$$

Since we assume that $\varepsilon<1$, it is enough to show that $\operatorname{Re}\left(f(I+\varepsilon f)^{-1}\right) \geq 0$.

$$
\begin{aligned}
\operatorname{Re}( & \left.f(I+\varepsilon f)^{-1}\right) \\
& =\frac{1}{2}\left(f(I+\varepsilon f)^{-1}+\left(I+\varepsilon f^{*}\right)^{-1} f^{*}\right) \\
& =\frac{1}{2}\left(I+\varepsilon f^{*}\right)^{-1}\left(\left(I+\varepsilon f^{*}\right) f+f^{*}(I+\varepsilon f)\right)(I+\varepsilon f)^{-1} \\
& =\frac{1}{2}\left(I+\varepsilon f^{*}\right)^{-1}\left(2 \operatorname{Re}(f)+2 \varepsilon|f|^{2}\right)(I+\varepsilon f)^{-1} \\
& \geq 0 .
\end{aligned}
$$

(3) As above,

$$
\begin{aligned}
\operatorname{Re}(I+ & \left.\left(f_{\varepsilon}-s I\right)\left(f_{\varepsilon}+s I\right)^{-1}\right) \\
= & I+\frac{1}{2}\left(\left(f_{\varepsilon}-s I\right)\left(f_{\varepsilon}+s I\right)^{-1}+\left(f_{\varepsilon}^{*}+s I\right)^{-1}\left(f_{\varepsilon}^{*}-s I\right)\right) \\
= & I+\frac{1}{2}\left(f_{\varepsilon}^{*}+s I\right)^{-1}\left(\left(f_{\varepsilon}^{*}+s I\right)\left(f_{\varepsilon}-s I\right)\right. \\
& \left.+\left(f_{\varepsilon}^{*}-s I\right)\left(f_{\varepsilon}+s I\right)\right)\left(f_{\varepsilon}+s I\right)^{-1} \\
= & I+\left(f_{\varepsilon}^{*}+s I\right)^{-1}\left(\left|f_{\varepsilon}\right|^{2}-s^{2} I\right)\left(f_{\varepsilon}+s I\right)^{-1} \\
= & \left(f_{\varepsilon}^{*}+s I\right)^{-1}\left(\left(f_{\varepsilon}^{*}+s I\right)\left(f_{\varepsilon}+s I\right)+\left|f_{\varepsilon}\right|^{2}-s^{2} I\right)\left(f_{\varepsilon}+s I\right)^{-1} \\
= & 2\left(f_{\varepsilon}^{*}+s I\right)^{-1}\left(\left|f_{\varepsilon}\right|^{2}+s \operatorname{Re} f_{\varepsilon}\right)\left(f_{\varepsilon}+s I\right)^{-1} .
\end{aligned}
$$


and the claim follows from the fact that $\operatorname{Re}\left(f_{\varepsilon}\right) \geq \varepsilon I$.

(4) We have for every $\varepsilon>0$,

$$
\begin{aligned}
f_{\varepsilon}-f & =(\varepsilon I+f)(I+\varepsilon f)^{-1}-f \\
& =((\varepsilon I+f)-f(I+\varepsilon f))(I+\varepsilon f)^{-1} \\
& =\varepsilon\left(I+f^{2}\right)(I+\varepsilon f)^{-1},
\end{aligned}
$$

so

$$
\mu_{t}\left(f_{\varepsilon}-f\right) \prec \prec \varepsilon \mu_{t}\left(I+f^{2}\right) \mu_{t}\left((I+\varepsilon f)^{-1}\right) .
$$

Since $\left\|(I+\varepsilon f)^{-1}\right\| \leq 1$, we get $\mu_{t}\left((I+\varepsilon f)^{-1}\right) \leq 1$ for every $t>0$. Also $I+f^{2} \in L^{p}(\mathscr{M}, \tau)$ for every $p>1$, so $\left\|f_{\varepsilon}-f\right\|_{p} \leq \varepsilon\left\|I+f^{2}\right\|_{p} \rightarrow 0($ as $\varepsilon \rightarrow 0)$. The proof is complete.

LEMMA 4. Let $a$ and $b$ be operators in $\overline{\mathscr{M}}$ with $a \geq 0, b \geq 0$, and let $P$ be $a$ projection that commutes with a. Then $\tau(a b) \geq \tau(P(a b) P)$.

Proof. To see this, notice that, since $P$ commutes with $a, P a P \leq a$, so $b^{1 / 2} P a P$ $b^{1 / 2} \leq b^{1 / 2} a b^{1 / 2}$, implying that $\tau\left(b^{1 / 2} P a P b^{1 / 2}\right) \leq \tau\left(b^{1 / 2} a b^{1 / 2}\right)$ and

$$
\begin{aligned}
\tau(P(a b) P) & =\tau(P(a b)) \\
& =\tau(P a P b)=\tau\left(b^{1 / 2} P a P b^{1 / 2}\right) \\
& \leq \tau\left(b^{1 / 2} a b^{1 / 2}\right)=\tau(a b) .
\end{aligned}
$$

The lemma is proved.

LEMMA 5. Let $S$ be a positive operator that commutes with $\left|f_{\varepsilon}\right|$ then

$$
\tau\left(S \operatorname{Re}\left(f_{\varepsilon}\right)\right) \leq \tau\left(S\left|f_{\varepsilon}\right|\right) .
$$

PROOF. Let $S \geq 0$ and assume that $S\left|f_{\varepsilon}\right|=\left|f_{\varepsilon}\right| S$. we have

$$
\begin{aligned}
\tau\left(S \operatorname{Re}\left(f_{\varepsilon}\right)\right) & =\tau\left(S\left(f_{\varepsilon}+f_{\varepsilon}^{*}\right) / 2\right) \\
& =\frac{1}{2}\left(\tau\left(S f_{\varepsilon}\right)+\overline{\tau\left(S f_{\varepsilon}\right)}\right) \\
& \leq\left|\tau\left(S f_{\varepsilon}\right)\right|
\end{aligned}
$$

Let $f_{\varepsilon}=u\left|f_{\varepsilon}\right|$ be the polar decomposition of $f_{\varepsilon}$. Since $S$ commutes with $\left|f_{\varepsilon}\right|$, we get $\left|\tau\left(S f_{\varepsilon}\right)\right|=\left|\tau\left(u\left|f_{\varepsilon}\right| S\right)\right|=\left|\tau\left(u S^{1 / 2}\left|f_{\varepsilon}\right| S^{1 / 2}\right)\right| \leq \tau\left(S\left|f_{\varepsilon}\right|\right)$. Thus the proof is complete. 
LEMMA 6. Let $A$ and $B$ be positive operators in $\mathscr{M}$ such that:

(i) $A^{-1}$ and $B^{-1}$ exists in $\mathscr{M}$;

(ii) $\tau(C A) \leq \tau(C B)$ for every $C$ that commutes with $B$;

then for every $C$ that commutes with $B, \tau\left(C B^{-1}\right) \leq \tau\left(C A^{-1}\right)$

Proof. Observe that $\tau\left(C B^{-1}\right)=\tau(\alpha \beta)$, where $\alpha=C^{1 / 2} B^{-1} A^{1 / 2}$ and $\beta=$ $A^{-1 / 2} C^{1 / 2}$. By Hölder's inequality,

$$
\begin{aligned}
\tau\left(C B^{-1}\right) & \leq \tau\left(|\alpha|^{2}\right)^{1 / 2} \tau\left(|\beta|^{2}\right)^{1 / 2}=\tau\left(\alpha^{*} \alpha\right)^{1 / 2} \tau\left(\beta^{*} \beta\right)^{1 / 2}=\tau\left(\alpha \alpha^{*}\right)^{1 / 2} \tau\left(\beta^{*} \beta\right)^{1 / 2} \\
& =\tau\left(C^{1 / 2} B^{-1} A^{1 / 2} A^{1 / 2} B^{-1} C^{1 / 2}\right)^{1 / 2} \tau\left(C^{1 / 2} A^{-1 / 2} A^{-1 / 2} C^{1 / 2}\right)^{1 / 2} \\
& =\tau\left(C^{1 / 2} B^{-1} A B^{-1} C^{1 / 2}\right)^{1 / 2} \tau\left(C A^{-1}\right)^{1 / 2} \\
& =\tau\left(B^{-1} C B^{-1} A\right)^{1 / 2} \tau\left(C A^{-1}\right)^{1 / 2} .
\end{aligned}
$$

Since $C$ commutes with $B$, the operator $B^{-1} C B^{-1}$ commutes with $B$ so we get by assumption that $\tau\left(B^{-1} C B^{-1}(A)\right) \leq \tau\left(B^{-1} C B^{-1}(B)\right)$, and therefore

$$
\tau\left(C B^{-1}\right) \leq \tau\left(C B^{-1}\right)^{1 / 2} \tau\left(C A^{-1}\right)^{1 / 2}
$$

which shows that $\tau\left(C B^{-1}\right) \leq \tau\left(C A^{-1}\right)$. The proof of the lemma is complete.

\section{Non-commutative variant of Kolmogorov Theorem}

In this section, our main goal is to present the proof of the inequality that will lead us to the Kolmogorov Theorem, that is the Hilbert transform for the case $p=1$.

THEOREM 2. Let $u \in \mathscr{M}$ with $u \geq 0$, and set $f=u+i \tilde{u}$. Then for every $s>0$,

$$
\tau\left(\chi_{(s, \infty)}(|f|)\right) \leq 4 \frac{\|u\|_{1}}{s} .
$$

PROOF. Our proof follows the presentation of Helson in [14] for the commutative case.

Let $u$ and $f$ be as in the statement of the Theorem 2, and fix $0<\varepsilon<1$. Set $f_{\varepsilon}$ as in Lemma 2. For $s \in(0, \infty)$ fixed, consider the following transformation on $\{z: \operatorname{Re}(z) \geq 0\}:$

$$
A_{s}(z)=1+\frac{z-s}{z+s} \quad \text { for all } z \in\{w: \operatorname{Re}(w) \geq 0\} .
$$

It can be checked that the part of the plane $\{z:|z| \geq s\}$ is mapped to the half disk $\{w: \operatorname{Re}(w) \geq 1\}$; this fact is very crucial in the argument of [14] for the commutative case. 
Note that $\sigma\left(f_{\varepsilon}\right)$ is a compact subset of $\{z: \operatorname{Re}(z) \geq \varepsilon\}$. By the analytic functional calculus for Banach algebras,

$$
A_{s}\left(f_{\varepsilon}\right)=I+\left(f_{\varepsilon}-s I\right)\left(f_{\varepsilon}+s I\right)^{-1} \in H^{\infty}
$$

and therefore (since $A_{s}$ is analytic)

$$
\Phi\left(A,\left(f_{\varepsilon}\right)\right)=A_{s}\left(\Phi\left(f_{\varepsilon}\right)\right)=A_{s}\left(\Phi(u)_{\varepsilon}\right) .
$$

Note that since $\Phi(u)$ is self-adjoint, so are $\Phi(u)_{\varepsilon}$ and $A_{s}\left(\Phi(u)_{\varepsilon}\right)$. We conclude from (3.1) that $\tau\left(I+\left(\Phi(u)_{\varepsilon}-s I\right)\left(\Phi(u)_{\varepsilon}+s I\right)^{-1}\right) \in \mathbb{R}$, and therefore

$$
\tau\left(\operatorname{Re}\left(I+\left(f_{\varepsilon}-s I\right)\left(f_{\varepsilon}+s I\right)^{-1}\right)\right)=\tau\left(A_{s}\left(\Phi(u)_{\varepsilon}\right)\right) .
$$

Set $P=\chi_{(s . x)}\left(\left|f_{\varepsilon}\right|\right)$. The projection $P$ commutes with $\left|f_{\varepsilon}\right|$ and we have

$$
\operatorname{Re}\left[I+\left(f_{\varepsilon}-s I\right)\left(f_{\varepsilon}+s I\right)^{-1}\right]=\left(f_{\varepsilon}^{*}+s I\right)^{-1}\left[2\left|f_{\varepsilon}\right|^{2}+2 s \operatorname{Re}\left(f_{\varepsilon}\right)\right]\left(f_{\varepsilon}+s I\right)^{-1} ;
$$

but since $\operatorname{Re}\left(f_{\varepsilon}\right) \geq \varepsilon I \geq 0$, we get

$$
2\left|f_{\varepsilon}\right|^{2}+2 s \operatorname{Re}\left(f_{\varepsilon}\right) \geq 2\left|f_{\varepsilon}\right|^{2},
$$

and hence

$$
\tau\left[\operatorname{Re}\left(I+\left(f_{\varepsilon}-s I\right)\left(f_{\varepsilon}+s I\right)^{-1}\right)\right] \geq \tau\left[2\left|f_{\varepsilon}\right|^{2}\left(f_{\varepsilon}+s I\right)^{-1}\left(f_{\varepsilon}^{*}+s I\right)^{-1}\right] .
$$

Applying Lemma 4 for $a=2\left|f_{\varepsilon}\right|$ and $b=\left(f_{\varepsilon}+s I\right)^{-1}\left(f_{\varepsilon}^{*}+s I\right)^{-1}$, we obtain

$$
\tau\left[\operatorname{Re}\left(I+\left(f_{\varepsilon}-s I\right)\left(f_{\varepsilon}+s I\right)^{-1}\right)\right] \geq \tau\left[2 P\left|f_{\varepsilon}\right|^{2}\left(f_{\varepsilon}+s I\right)^{-1}\left(f_{\varepsilon}^{*}+s I\right)^{-1}\right] .
$$

Note that $\left(f_{\varepsilon}+s I\right)^{-1}\left(f_{\varepsilon}^{*}+s I\right)^{-1}=\left(\left|f_{\varepsilon}\right|^{2}+2 s \operatorname{Re}\left(f_{\varepsilon}\right)+s^{2} I\right)^{-1}$.

Set

$$
\begin{aligned}
& A=\left(f_{\varepsilon}^{*}+s I\right)\left(f_{\varepsilon}+s I\right)=\left|f_{\varepsilon}\right|^{2}+2 s \operatorname{Re}\left(f_{\varepsilon}\right)+s^{2} I, \\
& B=\left|f_{\varepsilon}\right|^{2}+2 s\left|f_{\varepsilon}\right|+s^{2} I .
\end{aligned}
$$

It follows from Lemma 5 that if $C$ is a positive operator that commutes with $B$ then $\tau(C A) \leq \tau(C B)$.

Applying Lemma 6 to $A, B$ and $C=2 P\left|f_{\varepsilon}\right|^{2}$ (since $P$ commutes with $\left|f_{\varepsilon}\right|$, the operator $C$ commutes with $B$ ), we obtain from (3.4) that

$$
\tau\left[\operatorname{Re}\left(I+\left(f_{\varepsilon}-s I\right)\left(f_{\varepsilon}+s I\right)^{-1}\right)\right] \geq \tau\left(C A^{-1}\right) \geq \tau\left(C B^{-1}\right)
$$


and hence

$$
\tau\left[\operatorname{Re}\left(I+\left(f_{\varepsilon}-s I\right)\left(f_{\varepsilon}+s I\right)^{-1}\right)\right] \geq \tau\left[2 P\left|f_{\varepsilon}\right|^{2}\left(\left|f_{\varepsilon}\right|^{2}+s\left|f_{\varepsilon}\right|+s^{2} I\right)^{-1}\right] .
$$

If we denote by $E^{\left|f_{f}\right|}$ the spectral decomposition of $\left|f_{i}\right|$, then

$$
2 P\left|f_{\varepsilon}\right|^{2}\left(\left|f_{\varepsilon}\right|^{2}+2 s\left|f_{\varepsilon}\right|+s^{2} I\right)^{-1}=\int_{s}^{\infty} \frac{2 t^{2}}{t^{2}+2 s t+s^{2}} d E_{t}^{\left|f_{t}\right|} .
$$

Let

$$
\psi_{s}(t)=\frac{2 t^{2}}{t^{2}+2 s t+s^{2}} \quad \text { for } t \in[s, \infty)
$$

One can show that $\psi_{s}$ is increasing on $[s, \infty)$ so $\psi_{s}(t) \geq \psi_{s}(s)=\frac{1}{2}$ for $t \geq s$, and therefore

$$
\int_{s}^{\infty} \frac{2 t^{2}}{t^{2}+2 s t+s^{2}} d E_{t}^{\left|f_{s}\right|} \geq \frac{1}{2} P
$$

so we deduce from (3.5) that

$$
\tau\left[\operatorname{Re}\left(I+\left(f_{\varepsilon}-s I\right)\left(f_{\varepsilon}+s I\right)^{-1}\right)\right] \geq \frac{1}{2} \tau(P) .
$$

To finish the proof, recall from (3.2) that

$$
\tau\left[\operatorname{Re}\left(I+\left(f_{\varepsilon}-s I\right)\left(f_{\varepsilon}+s I\right)^{-1}\right)\right]=\tau\left[I+\left(\Phi(u)_{*}-s I\right)\left(\Phi(u)_{\varepsilon}+s I\right)^{-1}\right],
$$

so

$$
\tau(P) \leq 2 \tau\left[I+\left(\Phi(u)_{\varepsilon}-s I\right)\left(\Phi(u)_{\varepsilon}+s I\right)^{-1}\right]=2 \tau\left[2 \Phi(u)_{\varepsilon}\left(\Phi(u)_{\varepsilon}+s I\right)^{-1}\right] .
$$

But $\left(\Phi(u)_{\varepsilon}+s I\right)^{-1}=s^{-1}\left(\frac{\Phi(u)_{\varepsilon}}{s}+I\right)^{-1}$ has norm $\leq 1 / s$, hence

$$
\tau(P) \leq 4 \frac{\left\|u_{\varepsilon}\right\|_{1}}{s} .
$$

Now taking $\varepsilon \rightarrow 0$, we get from Lemma 3 (6) that $\left\|u_{*}\right\|_{1} \rightarrow\|u\|_{1}$ and $\left\|f_{\varepsilon}-f\right\|_{1}$ converges to zero. In particular, $\left(f_{\varepsilon}\right)$ converges to $f$ in measure. We obtain from [12, Lemma 3.4] that $\mu_{t}(f) \leq \liminf _{n \rightarrow \infty} \mu_{t}\left(f_{\varepsilon_{n}}\right)$ for each $t>0$ and $\varepsilon_{n} \rightarrow 0$. This implies that for every $s>0$ and every $t>0, \chi_{(s . \infty)}\left(\mu_{t}(f)\right) \leq \liminf _{n \rightarrow \infty} \chi_{(s . x)}\left(\mu_{t}\left(f_{\varepsilon_{n}}\right)\right)$. Hence 
by Fatou's lemma,

$$
\begin{aligned}
\tau\left(\chi_{(s . x)}(|f|)\right) & =\int_{0}^{1} \chi_{(s . \infty)}\left(\mu_{i}(|f|)\right) d t \\
& \leq \liminf _{n \rightarrow \infty} \int_{0}^{1} \chi_{(s . x)}\left(\mu_{t}\left(\left|f_{\varepsilon_{n}}\right|\right)\right) d t \\
& =\liminf _{n \rightarrow \infty} \tau\left(\chi_{(s . \infty)}\left(\left|f_{\varepsilon_{n}}\right|\right)\right) \\
& \leq \limsup _{n \rightarrow \infty}\left(4\left\|u_{\varepsilon_{n}}\right\|_{1} / s\right) .
\end{aligned}
$$

Hence $\tau\left(\chi_{(x, x)}(|f|)\right) \leq 4\|u\|_{1} / s$. The proof is complete.

As in the commutative case, Theorem 2 is used to extend the Hilbert transform to $L^{\prime}(\mathscr{M}, \tau)$. Recall that $L^{1 \chi}(\mathscr{M}, \tau)=\left\{a \in \overline{\mathscr{M}} ; \sup _{t>0} t \mu_{t}(a)<\infty\right\}$.

Set $\|a\|_{1 \cdot x}=\sup _{t>0} t \mu_{t}(a)$ for $a \in L^{1 \cdot x}(\mathscr{M}, \tau)$. As in the commutative case, \|\|$_{1 . x}$ is equivalent to a quasinorm in $L^{1 \cdot x}(\mathscr{M}, \tau)$, so there is a fixed constant $C$ such that, for every $a, b \in L^{1 \cdot x}(\mathscr{M}, \tau)$, we have $\|a+b\|_{1 . x} \leq C\left(\|a\|_{1, x}+\|b\|_{1 . x}\right)$.

For $u \in \mathscr{M}$, let $T u=u+i \tilde{u}$. The map $T$ is linear and Theorem 2 can be restated as follows:

For any $u \in \mathscr{H}$ with $u \geq 0$, we have $\|T u\|_{1 . x} \leq 4\|u\|_{1}$.

This implies that for $u \geq 0$,

$$
\|\tilde{u}\|_{1 . x} \leq C(4+1)\|u\|_{1}=5 C\|u\|_{1} .
$$

Now suppose that $u \in \mathscr{M}, u=u^{*}, u=u_{+}-u_{-}$and $\tilde{u}=\tilde{u}_{+}-\tilde{u}_{-}$. Then

$$
\|\tilde{u}\|_{1 . x} \leq C\left(\left\|\tilde{u}_{+}\right\|_{1 . x}+\left\|\tilde{u}_{-}\right\|_{1 . x}\right) \leq 5 C^{2}\|u\|_{1} .
$$

Similarly, if we require only $u \in \mathscr{M}$, we have $u=\operatorname{Re}(u)+i \operatorname{Im}(u)$ and by linearity, $\tilde{u}=\widehat{\operatorname{Re}(u)}+i \widehat{\operatorname{lm}(u)}$, and as above,

$$
\|\tilde{u}\|_{1, x} \leq 10 C^{3}\|u\|_{1} .
$$

We are now ready to define the extension $\mathscr{H}$ in $L^{1}(\mathscr{H}, \tau)$. If $u \in L^{1}(\mathscr{M}, \tau)$, let $\left(u_{n}\right)_{n \in \mathbb{N}}$ be a sequence in. $\mathscr{l}$ such that $\left\|u-u_{n}\right\|_{1} \rightarrow 0$ as $n \rightarrow \infty$. Then

$$
\left\|\tilde{u}_{n}-\tilde{u}_{n}\right\|_{1 . x} \leq 10 C^{3}\left\|u_{n}-u_{m}\right\|_{1},
$$

and since $\left\|u_{n}-u_{m}\right\|_{1} \rightarrow 0$ as $n, m \rightarrow \infty$, the sequence $\left(\tilde{u}_{n}\right)_{n}$ converges in $L^{1, \infty}(\mathscr{M}, \tau)$ to an operator $\tilde{u}$. This defines $\tilde{u}$ for $u \in L^{1}(\mathscr{M}, \tau)$. This definition can be easily checked to be independent of the sequence $\left(\tilde{u}_{n}\right)_{n}$ and agree with the conjugation operator defined for $p>1$.

Letting $n \rightarrow \infty$ in the inequality $\left\|\tilde{u}_{n}\right\|_{1 . \infty} \leq 10 C^{3}\left\|u_{n}\right\|_{1}$, we obtain the following theorem $\left(H^{1 \cdot x}\right.$ denotes the closure of $H^{\infty}$ in $L^{1 \cdot x}(\mathscr{M}, \tau)$ ): 
THEOREM 3. There is a unique extension of $\mathscr{H}$ from $L^{1}(\mathscr{M}, \tau)$ into $L^{1, x}(\mathscr{M}, \tau)$ with the following property: $u+i \tilde{u} \in H^{1 . x}$ for all $u \in L^{1}(\mathscr{M}, \tau)$, and there is a constant $K$ such that $\|\tilde{u}\|_{1 . x} \leq K\|u\|_{1}$ for all $u \in L^{1}(\mathscr{M} . \tau)$.

COROLlaRY 1. For any $p$ with $0<p<1$ there exists a constant $K_{p}$, such that

$$
\|\tilde{u}\|_{p} \leq K_{p}\|u\|_{1} \text { for all } u \in L^{1}(\mathscr{H} \cdot \tau) .
$$

PROOF. It is enough to show that such a constant exists for $u \in \mathscr{M}, u \geq 0$. Recall that for $u \in \mathscr{M}$, the distribution $\lambda_{s}(u)$ equals $\tau\left(\chi_{(s . x)}(u)\right)$.

Let $F(s)=1-\lambda_{s}(u)=\tau\left(\chi_{(0 . s)}(u)\right)$. Assume that $\|u\|_{1} \leq 1$. From Theorem 2,

$$
1-F(s) \leq \frac{4}{s}\|u\|_{1} \leq \frac{4}{s} .
$$

Note that $F$ is a non-increasing right continuous function and for $p>0$,

$$
\tau\left(|\tilde{u}|^{p}\right)=\int_{0}^{1} \mu_{t}(|\tilde{u}|)^{p} d t=\int_{0}^{\infty} s^{p} d F(s) \leq 1+\int_{1}^{\infty} s^{p} d F(s) .
$$

If $A$ is a point of continuity for $F(A>1)$, then

$$
\int_{1}^{A} s^{p} d F(s)=\left[s^{p}(F(s)-1)\right]_{1}^{A}+p \int_{1}^{A}(1-F(s)) s^{p-1} d s .
$$

Since $1-F(s) \leq 4 / s$, we get that both $\left[s^{p}(F(s)-1)\right]_{1}^{A}$ and $\int_{1}^{A}(1-F(s)) s^{p-1} d s$ are bounded for $0<p<1$, that is, $\int_{0}^{1} \mu_{t}(|\tilde{u}|)^{p} d t$ has bound independent of $u$.

The Riesz projection $\mathscr{R}$ can now be defined as in the commutative case: for every $a \in L^{p}(\mathscr{M}, \tau),(1 \leq p \leq \infty)$,

$$
\mathscr{R}(a)=\frac{1}{2}(a+i \tilde{a}+\Phi(a)) .
$$

From Theorem 1, one can easily verify that $\mathscr{R}$ is a bounded projection from $L^{p}(\mathscr{M}, \tau)$ onto $H^{p}$ for $1<p<\infty$. In particular $H^{p}$ is a complemented subspace of $L^{p}(\mathscr{M}, \tau)$.

For $p=1$, Theorem 3 shows that $\mathscr{R}$ is bounded from $L^{i}(\mathscr{H}, \tau)$ into $H^{1 . x}$.

Our next result gives a sufficient condition on an operator $a \in L^{1}(\mathscr{M}, \tau)$ so that its conjugate $\tilde{a}$ belongs to $L^{1}(\mathscr{M}, \tau)$.

THEOREM 4. There exists a constant $K$ such that for every positive $a \in \mathscr{M}$.

$$
\|\tilde{a}\|_{1} \leq K \tau\left(a \log ^{+} a\right)+K .
$$


Proof. Let $C$ be the absolute constant such that $\|\tilde{a}\|_{p} \leq C p q\|a\|_{p}$ for all $a \in$ $L^{p}(. \mathscr{U} . \tau), 1<p<\infty$ and $1 / p+1 / q=1$. The conclusion of the theorem can be deduced as a straightforward adjustment of the commutative case in [31, p. 119]; we will present it here for completeness.

Let $a \in \mathscr{H}$; we will assume first that $a \geq 0$. Let $\left(e_{t}\right)_{t}$ be the spectral decomposition of $a$. For each $k \in \mathbb{N}$, let $P_{k}=\chi_{\left[2^{k}+.2^{k},\right.}(a)$ be the spectral projection relative to $\left[2^{k-1} \cdot 2^{k}\right)$. Define $a_{k}=a P_{k}$ for $k \geq 1$ and $a_{0}=a \chi_{(0.1)}(a)$. Clearly $a=\sum_{k=0 !}^{\infty} a_{k}$ in $L^{p}(. \mathscr{H} . \tau)$ for every $1<p<\infty$. By linearity, $\tilde{a}=\sum_{k=0}^{\infty} \tilde{a}_{k}$. For every $k \in \mathbb{N}$, $\left\|\tilde{a}_{k}\right\|_{1} \leq\left\|\tilde{a}_{k}\right\|_{p} \leq C p^{2}(p-1)^{-1}\left\|a_{k}\right\|_{p}$. Since $a_{k} \leq 2^{k} P_{k}$, we get for $1<p<2$,

$$
\left\|\tilde{a}_{k}\right\|_{1} \leq 4 C \frac{1}{p-1} 2^{k} \tau\left(P_{k}\right)^{\frac{1}{1}} \text {. }
$$

If we set $p=1+1 /(k+1)$ and $\epsilon_{k}=\tau\left(P_{k}\right)$, we have

$$
\left\|\tilde{a}_{k}\right\|_{1} \leq 4 C(k+1) 2^{k} \epsilon_{k}^{\frac{k-1}{k-2}} .
$$

Taking the summation over $k$,

$$
\|\tilde{a}\|_{1} \leq \sum_{k=0}^{x} 4 C(k+1) 2^{k} \epsilon_{k}^{\frac{k-1}{k-2}} .
$$

We note as in [31] that if $J=\left\{k \in \mathbb{N} ; \epsilon_{k} \leq 3^{-k}\right\}$ then

$$
\sum_{k \in J} 4 C(k+1) 2^{k} \epsilon_{k}^{\frac{k-1}{k-2}} \leq \sum_{k=0}^{\infty} 4 C(k+1) 2^{k}\left(3^{-k}\right)^{\frac{k+1}{k-2}}=\alpha<\infty .
$$

On the other hand. for $k \in \mathbb{N} \backslash J, \epsilon_{k}^{\frac{k-1}{k-2}} \leq \epsilon_{k} 3^{\frac{k}{k+2}} \leq \beta \epsilon_{k}$ where $\beta=\sup _{k} 3^{\frac{k}{k+2}}$. So we get

$$
\begin{aligned}
\|\tilde{a}\|_{1} & \leq \alpha+4 C \beta \sum_{k=0}^{\infty}(k+1) 2^{k} \epsilon_{k} \\
& \leq \alpha+4 C \beta\left(\epsilon_{0}+4 \epsilon_{1}\right)+4 C \beta \sum_{k \geq 2}(k+1) 2^{k} \epsilon_{k} .
\end{aligned}
$$

Since for $k \geq 2, k+1 \leq 3(k-1)$, we get

$$
\|\tilde{a}\|_{1} \leq \alpha+16 C \beta+24 C \beta \sum_{k \geq 2}(k-1) 2^{k-1} \epsilon_{k} .
$$

To complete the proof, notice that for $k \geq 2$,

$$
\begin{aligned}
(k-1) 2^{k-1} \epsilon_{k} & =\int_{2^{k-1}}^{2^{k}}(k-1) 2^{k-1} d \tau\left(e_{t}\right) \\
& \leq \int_{2^{k-1}}^{2^{k}} \frac{t \log t}{\log 2} d \tau\left(e_{1}\right) .
\end{aligned}
$$


Hence by setting $K=\max \{\alpha+16 C \beta, 24 C \beta / \log 2\}$, we get:

$$
\|\tilde{a}\|_{1} \leq K+K \tau\left(a \log ^{+}(a)\right) .
$$

The proof is complete.

\section{Concluding remarks}

From Theorem 4, one can deduce that if $a \in L^{\prime}(\mathscr{M}, \tau)$ is such that $a \geq 0$ and $a \log ^{+}(a)$ belongs to $L^{1}(\mathscr{M}, \tau)$ then $\tilde{a} \in L^{1}(\mathscr{M}, \tau)$. It is natural to try to characterize the operators $u \in L^{1}(\mathscr{M}, \tau)$ for which $\tilde{u} \in L^{1}(\mathscr{M}, \tau)$. For the classical Hardy spaces on $\mathbb{T}$ and $\mathbb{R}^{n}$, a complete characterization was obtained by Davis in [5] using signed decreasing functions. Later, Davis's result was generalized by Lancien for weak*-Dirichlet algebras (see [18]) so the following question seems to be of interest:

PROBLEM 1. Is it possible to characterize all operators with conjugate in $L^{1}(\mathscr{M}, \tau)$ ?

Let us finish with the following open question that arises from the classical case and the topic of this paper: it is a well-known result of Bourgain [3] that $L^{1}(\mathbb{T}) / H^{1}(\mathbb{T})$ is of cotype 2 and later Lancien [17] proved that a similar result holds for $L^{1} / H^{1}$ associated with weak*-Dirichlet algebras.

PROBLEM 2. Is $L^{1}(\mathscr{M}, \tau) / H^{1}(\mathscr{M}, \tau)$ of cotype 2? (or merely of finite cotype?)

It should be noted that the theory of conjugate functions and the boundedness of the Riesz projection were very crucial in the proof given by Bourgain [3] for the classical case and Lancien [17] for the setting of weak*-Dirichlet algebras.

\section{Acknowledgements}

Parts of the work reported here were done during the author's visit at the Mathematical Sciences Research Institute (MSRI) in Berkeley, CA. The author is grateful to the organizers of the Special Semester on Convex Geometry for financial support. 


\section{References}

[1] W. Arveson, 'Analyticity in operator algebras', Amer. J. Math. 89 (1967), 578-642.

[2] N. Asmar, E. Berkson and T. A. Gillespie, 'Representation of groups with ordered duals and generalized analyticity', J. Funct. Anal. 90 (1990), 206-235.

[3] J. Bourgain, 'Bilinear forms on $H^{x}$ and bounded bianalytic functions', Trans. Amer. Math. Soc. 286 (1984), 313-337.

[4] V. I. Chilin and F. A. Sukochev, 'Symmetric spaces on semifinite von Neumann algebras', Soviet Math. Dokl. 42 (1992), 97-101.

[5] B. Davis, 'Hardy spaces and rearrangements', Trans. Amer. Math. Soc. 261 (1980), 211-233.

[6] A. Devinatz, 'Conjugate function theorems for Dirichlet algebras', Rev. Un. Mat. Argentina 23 (1966/67), 3-30.

[7] J. Diestel, Sequences and series in Banach spaces, Grad. Texts in Math. 92 (Springer Verlag, New York, 1984).

[8] P. G. Dodds, T. K. Dodds and B. de Pagter, 'Non-commutative Banach function spaces', Math. Z. 201 (1989), 583-597.

[9] - 'Remarks on non-commutative interpolation', Proc. Centre Math. Anal. Austral. Nat. Univ. 24 (1989).

[10] - 'Non-commutative Köthe duality', Trans. Amer. Math. Soc. 339 (1993), 717-750.

[11] P. G. Dodds, T. K. Dodds. B. de Pagter and F. A. Sukochev, 'Lipschitz continuity of the absolute value and Riesz projections in symmetric operator spaces', J. Funct. Anal. 148 (1997), 28-69.

[12] T. Fack and H. Kosaki, 'Genaralized s-numbers of $\tau$-measurable operators', Pacific J. Math. 123 (1986), 269-300.

[13] T. A. Gillepsie, E. Berkson and P. S. Muhly, 'Abstract spectral decomposition guarranted by Hilbert transform', Proc. London Math. Soc. 53 (1986), 489-517.

[14] H. Helson, Harmonic Analysis (Addison-Wesley, London, Amsterdam, Sydney and Tokyo, 1983).

[15] I. Hirschman, Jr and R. Rochberg, 'Conjugate function theory in weak*-Dirichlet algebras', $J$. Funct. Anal. 16 (1974), 359-371.

[16] S. Kawamura and J. Tomiyana, 'On subdiagonal algebras associated with flows in operator algebras', J. Math. Soc. Japan 29 (1977), 73-90.

[17] F. Lancien, 'Generalization of Bourgain's theorem about $L^{1} / H^{1}$ for weak*-Dirichlet algebras', Houston J. Math. 20 (1994), 47-61.

[18] - 'Distribution of functions in abstract $H^{1}$, lllinois J. Math. 39 (1995), 181-186.

[19] J. Lindenstrauss and L. Tzafriri, Classical Banach spaces II, Modern Surveys In Mathematics 97 (Springer-Verlag, Berlin-Heidelberg-New York, 1979).

[20] M. Marsalli, 'Non-commutative $H^{2}$ spaces', Proc. Amer. Math. Soc. 125 (1997), 779-784.

[21] M. Marsalli and G. West. 'Non-commutative $H^{p}$ spaces', preprint.

[22] M. McAsey, P. S. Muhly and K. S. Saito, 'Non-self adjoint crossed products (invariant subspaces and maximality)', Trans. Amer. Math. Soc. 248 (1979), 381-410.

[23] E. Nelson, 'Notes on non-commutative integration', J. Funct. Anal. 15 (1974), 103-116.

[24] K. S. Saito, 'On non-commutative Hardy spaces associated with flows on finite von-Neumann algebras', Tôhoku Math. J. 29 (1977), 585-595.

[25] _- 'A note on invariant subspaces for finite maximal subdiagonal algebras', Proc. Amer. Math. Soc. 77 (1979), 348-352.

[26] I. E. Segal, 'A non-commutative extension of abstract integration', Ann. of Math. 57 (1953), $401-457$.

[27] M. Takesaki, Theory of operator Algebras I (Springer-Verlag, New-York, Heidelberg, Berlin, 1979).

[28] Q. Xu, 'Analytic functions with values in lattices and symmetric spaces of measurable operators', 
Math. Proc. Cambridge Philos. Soc. 109 (1991), 541-563.

[29] K. Yosida, Functional analysis (Springer-Verlag, Berlin, New-York. 1980).

[30] L. Zsido, 'On spectral subspaces associated to locally compact abelian groups of operators', Ads: Math. 36 (1980), 213-276.

[31] A. Zygmund, Trigonometric series, volume II (Cambridge University Press. London. 1959).

Department of Mathematics and Statistics

Miami University

Oxford

$\mathrm{OH} 45056$

e-mail: randrin@muohio.edu 